\title{
Hair analysis for the biomonitoring of pesticide exposure: comparison with blood and urine in a rat model
}

\author{
Brice M. R. Appenzeller ${ }^{1} \cdot$ Emilie M. Hardy $^{1} \cdot$ Nathalie Grova $^{1} \cdot$ Caroline Chata $^{1}$. \\ François Faÿs $^{1,2} \cdot$ Olivier Briand $^{3} \cdot$ Henri Schroeder $^{4} \cdot$ Radu-Corneliu Duca $^{1}$
}

Received: 8 November 2016 / Accepted: 6 December 2016 / Published online: 23 December 2016

(c) The Author(s) 2016. This article is published with open access at Springerlink.com

\begin{abstract}
Urine and plasma have been used to date for the biomonitoring of exposure to pollutants and are still the preferred fluids for this purpose; however, these fluids mainly provide information on the short term and may present a high level of variability regarding pesticide concentrations, especially for nonpersistent compounds. Hair analysis may provide information about chronic exposure that is averaged over several months; therefore, this method has been proposed as an alternative to solely relying on these fluids. Although the possibility of detecting pesticides in hair has been demonstrated over the past few years, the unknown linkage between exposure and pesticides concentration in hair has limited the recognition of this matrix as a relevant tool for assessing human exposure. Based on a rat model in which there was controlled exposure to a mixture of pesticides composed of lindane, $\beta$-hexachlorocyclohexane, $\beta$-endosulfan, $\mathrm{p}, \mathrm{p}^{\prime}$-DDT,
\end{abstract}

Electronic supplementary material The online version of this article (doi:10.1007/s00204-016-1910-9) contains supplementary material, which is available to authorized users.

Brice M. R. Appenzeller

brice.appenzeller@lih.lu

1 Human Biomonitoring Research Unit, Department of Population Health, Luxembourg Institute of Health, 29 Rue Henri Koch, 4354 Esch-sur Alzette, Luxembourg

2 Competence Center in Methodology and Statistics, Luxembourg Institute of Health, 29 Rue Henri Koch, 4354 Esch-sur Alzette, Luxembourg

3 French Ministry of Agriculture, Agrifood, and Forestry, Paris, France

4 Unit Research Animal and Functionality of Animal Products (URAFPA), French National Institute for Agricultural Research (INRA) UC340, University of Lorraine, Nancy, France $\mathrm{p}, \mathrm{p}^{\prime}$-DDE, dieldrin, pentachlorophenol, diazinon, chlorpyrifos, cyhalothrin, permethrin, cypermethrin, propiconazole, fipronil, oxadiazon, diflufenican, trifluralin, carbofuran, and propoxur, the current work demonstrates the association between exposure intensity and resulting pesticide concentration in hair. We also compared the results obtained from a hair analysis to urine and plasma collected from the same rats. Hair, blood, and urine were collected from rats submitted to 90-day exposure by gavage to the aforementioned mixture of common pesticides at different levels. We observed a linear relationship between exposure intensity and the concentration of pesticides in the rats' hair $\left(R_{\text {Pearson }} 0.453-0.978, p<0.01\right)$. A comparison with results from urine and plasma samples demonstrated the relevance of hair analysis and, for many chemicals, its superiority over using fluids for differentiating animals from different groups and for re-attributing animals to their correct groups of exposure based on pesticide concentrations in the matrix. Therefore, this study strongly supports hair analysis as a reliable tool to be used during epidemiological studies to investigate exposure-associated adverse health effects.

Keywords Hair analysis · Plasma · Urine · Pesticide · Biomonitoring $\cdot$ Exposure

\section{Introduction}

Both the adverse effects of pesticides and the ubiquity of human exposure to these chemicals have been documented by an increasing number of data sets (Ntzani et al. 2013). Although different approaches can be used to assess exposure, biomonitoring (detecting chemicals and/or their metabolites in biological matrices) often remains as the preferred approach because it offers the advantage of 
integrating all of the possible sources and routes of exposure and of representing the internal dose from exposure. Correctly and accurately assessing the level of exposure to organic pollutants such as pesticides, however, remains challenging. Indeed, mainly because of logistical and financial constraints, an exposure assessment most often relies on a single biological sample per individual, typically blood or urine, the latter being generally preferred because urine is collected in a noninvasive manner (Esteban and Castaño 2009). Nevertheless, for most compounds, rapid elimination from urine between successive exposure episodes results in short temporal windows of detection [defined as the timeframe within which a compound can be detected since exposure occurred (i.e., before sampling takes place and before the measured concentration is determined)] and in high variability in urinary concentration of chemicals (Attflied et al. 2014; Aylward et al. 2014). For instance, the within-subjects variability in the concentration of organophosphorus and pyrethroid urinary metabolites measured in children from the Seattle, WA, area followed over 1 year was reported to be 2-11 times higher than the between-individual variability (Attflied et al. 2014). Moreover, it was demonstrated that a single urine sample was clearly insufficient to consistently categorize children's exposure into quartiles (Attflied et al. 2014). The variability associated with chemical concentrations in biological fluids, especially for short half-life chemicals, increases the risk of misclassification of individuals with regard to their exposure levels, and this results in a dramatic loss in statistical power in a study of associated adverse health effects.

To overcome the limitations associated with conventional biological matrices, novel approaches based on alternative matrices such as hair have been suggested. For instance, a paper (Appenzeller and Tsatsakis 2012) reviewed several publications that reported the possibility of detecting organic pollutants from different chemical classes in hair, reflecting individuals' environmental or occupational exposure. Hair samples can be collected in a noninvasive manner and can be easily stored. However, the main advantage associated with this matrix lies in the possibility to reach extended windows of detection that may represent up to several months of exposure, depending on the length of the sample. Contrary to biological fluids such as urine and blood, the concentration of chemicals detected in hair is not influenced by short-term variations in the exposure. Instead, the concentration corresponds to an individual's average level of exposure, which is the most relevant information for investigating possible linkages with biological effects. Although hair was reportedly used during the 1950s through 1970s to analyze for metals and was employed during the 1970s through the 1980s to determine drugs of abuse, using this matrix for detecting organic pollutants has been delayed by several limitations. For instance, there was a lack of sensitive analytical methods to sufficiently and properly monitor environmental exposure (Appenzeller 2015). This issue has, however, been resolved because considerable efforts were made during the past few years that now enable the methods to reach sensitivity levels that stand between 1000 and 10,000 times below those obtained 10 years ago (Appenzeller 2015; Salquebre et al. 2012). The influence of pigmentation on the incorporation of chemicals in hair has also been investigated, but a consistent conclusion has not yet been reached on this topic. For instance, although some studies suggested that melanin influences the concentration of some illicit and medical drugs in human and animal hair (Appenzeller and Tsatsakis 2012), no or limited influence of pigmentation was observed for ethylglucuronide, phase II metabolite of ethanol (Appenzeller et al. 2007a; Kharbouche et al. 2010), and for metabolites of polycyclic aromatic hydrocarbons (Grova et al. 2013). The main criticism of using hair analysis for the biomonitoring of pollutant exposures involves the representativeness of the level of exposure. Because qualitative results (presence or absence of drugs) are often sufficient in medico-legal contexts to prove consumption, the linkage between intake intensity and resulting concentration in hair has been poorly investigated in the past. The proportional relationship has, however, been demonstrated between ethanol consumption and the concentration of its metabolite ethyl glucuronide in hair for both humans (Appenzeller et al. 2007a) and rats (Kharbouche et al. 2010). Regarding organic pollutants, promising results have already demonstrated that rabbits exposed to a high dose of pesticides presented higher concentrations of chemicals in hair than those exposed to a low dose for organophosphates (Maravgakis et al. 2012; Margariti and Tsatsakis 2009; Tutudaki et al. 2003) and cypermethrin (pyrethroid) (Kavvalakis et al. 2014). Nevertheless, studies investigating the linkage between exposure and pesticide concentration in hair that cover a wide range of concentration levels of several chemicals are still needed so hair analysis will be recognized as a reliable marker for assessing the intensity of exposures.

In the current study, we investigated the linkage between the exposure level and the resulting concentration of pesticides and their metabolites in hair. We used rats during the study and controlled the exposure to a mixture of 19 pesticides from different chemical classes at eight different doses over a 90-day period. We compared the association between exposure level and the concentration of chemicals in hair collected at the end of the experiment to plasma and urinary concentrations collected from the same animals. For the three matrices, we also assessed the difference in pesticide concentrations between the different groups of exposure and the possibility to back determine the animals' level of exposure on the basis of pesticide concentrations in hair, urine, and plasma. 


\section{Materials and methods}

\section{Animal experimentation}

\section{Animal housing}

Sixty-eight bicolor (white and black hair) female LongEvans rats (180-200 g, Elevage Janvier, St. Berthevin, France) were housed two per cage in a regulated environment (temperature $22 \pm 3{ }^{\circ} \mathrm{C}$; relative humidity $55 \pm 10 \%$ ) under a reversed light-dark cycle (lights on from 7:00 p.m. to 7:00 a.m.). Food (Teklad Global Diet 2016, Harlan, Gannat, France) and water were available ad libitum. The rats were acclimatized to the animal facility for 2 weeks before the experiment began. To minimize the external contamination of hair by pesticides from urine excretion, special bedding [with a high water-binding capacity (372\%), Lignocel $3 / 4$, Harlan, Gannat, France] was replaced twice per week. Moreover, to evaluate the potential external contamination because of urine excretion, the bedding of the highest level exposed group was placed into a sentinel cage that containing four non-treated rats, which were analyzed at the end of the experiment. Feces were removed prior to placing the sentinel rats on the soiled bedding, which was replaced twice per week for the entire duration of the experiment. The analysis of the sentinel rats' hair did not demonstrate contamination of hair because of the bedding material. The procedures applied were in compliance with the rules provided by the European Union (2010/63/EU) and were approved and supervised by the Institutional Ethics Committee of the University of Lorraine (authorization number B 54-547-13).

\section{Animal treatment}

Eight rats were randomly assigned to each of the experimental groups. A low-calorie water gel that contained the pesticides was administered via gavage to these rats three times per week for 90 days. The doses of the pesticides mixture used for exposure were 4, 10, 20, 40, 100, 200, and $400 \mu \mathrm{g} / \mathrm{kg}$ of body weight. The exposure range was set as follows: The lowest dose was the lowest level allowing the detection of pesticides in hair after a 90-day exposure, based on preliminary experiments not detailed here. Testing lower levels would not have been relevant because some compounds would not be detected anymore (analytical limitation). In addition, for some other compounds, no differences were detected in the current experiment between the lowest level of exposure and the background exposure of the controls. The highest dose was set according to toxicity of compounds, which corresponded to $1 / 20$ of the lowest LD50 (carbofuran). The animals were weighed before each administration in order to adapt the amount of pesticidecontaining mixture to the animals' weight.

\section{Pesticide gavage mixture}

Pesticide mixture stock solution was prepared in ethanol every 2 weeks. Gels were prepared by mixing hot HydraGel and MediGel Sucralose (1/1, v/v, Bio Services, Uden, Netherlands), pouring the mixture into aluminum molds, and then allowing it to cool at room temperature. The gels were supplemented with the appropriate volume of pesticide mixture stock solution. Ethanol was allowed to dry at room temperature $\left(\sim 25^{\circ} \mathrm{C}\right)$ until complete evaporation (i.e., minimum of $4 \mathrm{~h}$ ). A second layer of gel was then deposited to trap the pesticides inside the gel. The control rats were fed with the same gel that was free of the pesticides. The optimal evaporation time was previously determined on gels supplemented with ethanol. The ethanol content was then assessed at different time points by using a headspace sampler coupled gas chromatography-mass spectrometry (GC-MS) instrument (Agilent Technologies, Diegem, Belgium).

\section{Samples collection}

The animals were shaved before the experiment began to ensure that the hair collected at the end of the study accurately reflected the 90-day period of exposure. White and black hairs were collected separately, placed in aluminum foil, and stored at $-20{ }^{\circ} \mathrm{C}$ until analysis. Blood was collected in EDTA tube from the tail vein, $3 \mathrm{~h}$ after oral administration on Day 90. Each sampling (500-750 $\mu \mathrm{L})$ was immediately centrifuged at $5000 \times g$ for $3 \mathrm{~min}$ at room temperature, and plasma was separated and stored at $-80{ }^{\circ} \mathrm{C}$ before analysis. For urine collection, the rats were placed in individual metabolic cages (Type 304 stainless steel, Techniplast, Zwaag, Netherlands) immediately after gavage for $24 \mathrm{~h}$ (from Day 88 to Day 89). The urine was collected in refrigerated tubes over a 24 -h period and was weighed before storage at $-20{ }^{\circ} \mathrm{C}$.

\section{Pesticides and analysis}

The list of pesticides to which the animals were exposed included compounds from different chemical classes (see Table S1), covering a wide range of different physicochemical properties to confirm that the association between exposure and the resulting concentration in hair was not limited to a specific category of chemicals (Chata et al. 2016). The list of pesticides included chemicals classically investigated in humans (e.g., organochlorines, organophosphates, pyrethroids) in order to allow for a comparison with data obtained from the literature for humans, as well as 
pesticides that have been investigated less or not at all in humans.

Depending on the compounds, only parent pesticides, only metabolites, or both parent and metabolites were analyzed in the biological matrices. Details about the target chemicals are provided in Table S1. The chemicals were analyzed as previously described (Chata et al. 2016; Hardy et al. 2015). Despite the different nature of the biological matrices analyzed (liquid vs. solid), method protocols were developed to ensure similarity so they could be reliably compared to the information obtained from each matrix, thereby ensuring that differences were not likely attributable to analytical bias. Moreover, the sensitivity of the methods used proved to be quite satisfactory with regard to the literature (comparable to the best performances in the field); this ensured that the differences between matrices were not because of a lack of sensitivity (Hardy et al. 2015). Because analytical background noise was absent from the chromatograms because tandem mass spectrometry was used, the approaches based on background noise for determining the limit of detection (LOD) were not applicable. The LOD was therefore determined as the lowest concentration that was detected in the samples analyzed during this study. Selectivity was ensured by analyte retention time and by the quantification transition to confirmation transition ratio that had to be lower than the $20 \%$ difference from the ratio obtained with standard compounds. The LOD ranged from $0.02 \mathrm{pg} / \mathrm{mg}$ for $\beta$-endosulfan to $2.7 \mathrm{pg} / \mathrm{mg}$ for dichlorodiphenyltrichloroethane (p, $\mathrm{p}^{\prime}$-DDT) in hair, from $0.2 \mathrm{pg} / \mathrm{mL}$ for trifluralin to $13.6 \mathrm{ng} / \mathrm{mL}$ for 2-isopropoxyphenol (2-IPP) in urine, and from $2.4 \mathrm{pg} / \mathrm{mL}$ for $\beta$-endosulfan to $679 \mathrm{pg} / \mathrm{mL}$ for $\mathrm{p}, \mathrm{p}^{\prime}$-DDT in plasma. Because the analysis of parent pesticides and metabolites was conducted on the same hair sample, the addition of parent organophosphate isotope-labeled analog standards was not possible because their degradation into non-labeled dialkyl phosphate (DAP) during the analytical procedure would have hindered the analysis of DAP due to exposure. In hair, only organophosphate metabolites (not parents) were therefore quantified, and only qualitative results were obtained for parents.

\section{Statistical analysis}

The association between the level of exposure and the analyte concentration in the matrix (i.e., hair, urine, and plasma) was assessed by the Pearson product-moment correlation coefficient $\left(R_{\text {Pearson }}\right)$. The global tendency to present different analyte concentrations in the matrix for different levels of exposure was assessed by the Spearman's correlation coefficient on ranks $\left(R_{\text {Spearman }}\right)$. The values calculated for slope, $R_{\text {Pearson }}$, and $R_{\text {Spearman }}$ only took into account samples with detected concentrations and included control animals when the target compounds were detected in the samples collected from them (e.g., lindane).

Inter-group differences in analyte concentration in the matrices were furthermore tested with a $t$ test or a MannWhitney Rank Sum test when normality (Shapiro-Wilk) or equal variance test failed (SigmaPlot 12.0). For the intergroup difference statistical testings, the value of $1 / 2 \mathrm{LOD}$ was attributed to the samples with a non-detected concentration. The differences between groups presented in Figs. S2 through S4 were tested as follows: top-down, the highest exposure group ( $400 \mu \mathrm{g} / \mathrm{kg})$ was compared to the group just below $(200 \mu \mathrm{g} / \mathrm{kg})$. If a significant difference was observed, then it was marked with brackets, and the difference was then tested between the 200 and the $100 \mu \mathrm{g} / \mathrm{kg}$ groups. If no significant difference was observed between the 400 and the $200 \mu \mathrm{g} / \mathrm{kg}$ groups, then the $400 \mu \mathrm{g} / \mathrm{kg}$ group was first compared with the $100 \mu \mathrm{g} / \mathrm{kg}$ group, and then with groups of lower exposure if no difference was observed. The procedure was then repeated all along the groups and was then re-applied down-top (starting with the control group) in order to test all the groups.

To investigate to what extent the results obtained from hair, urine, and plasma analyses may help to accurately categorize individuals according to their level of exposure, a reverse classification analysis (RCA) was conducted based on the approach described for humans (Attflied et al. 2014). For a set of five randomly selected animals, three ascending sorts were conducted based on pesticide concentrations in hair, urine, and plasma, respectively, and then were compared with a classification according to the animals' level of exposure used as a reference. For each matrix, one point was added if matrix-based classification was correct (cases of equality in doses were also considered), and no point was added if it was different. The procedure was reiterated 10,000 times, and the percentage of correct classification for each matrix and each compound is presented in Fig. 2 . RCA was not conducted for chemicals detected in less than four groups.

\section{Results}

For all of the chemicals (parent and metabolites) that were detected in hair or urine, the concentration in the matrix was significantly associated with the level of exposure $(p<0.01)$. In plasma, only diethyl phosphate (DEP) and diethylthiophosphate (DETP) were not significantly associated with the level of exposure, although these metabolites were always present. Slopes (linear fit) of analyte concentration in hair $(\mathrm{pg} / \mathrm{mg})$ versus level of exposure varied from 1.11 for trifluralin to 612 for $\beta$-hexachlorocyclohexane $(\beta-\mathrm{HCH})($ Table 1$)$. Considerable differences in the slope "concentration in the matrix" versus the "level of exposure" 


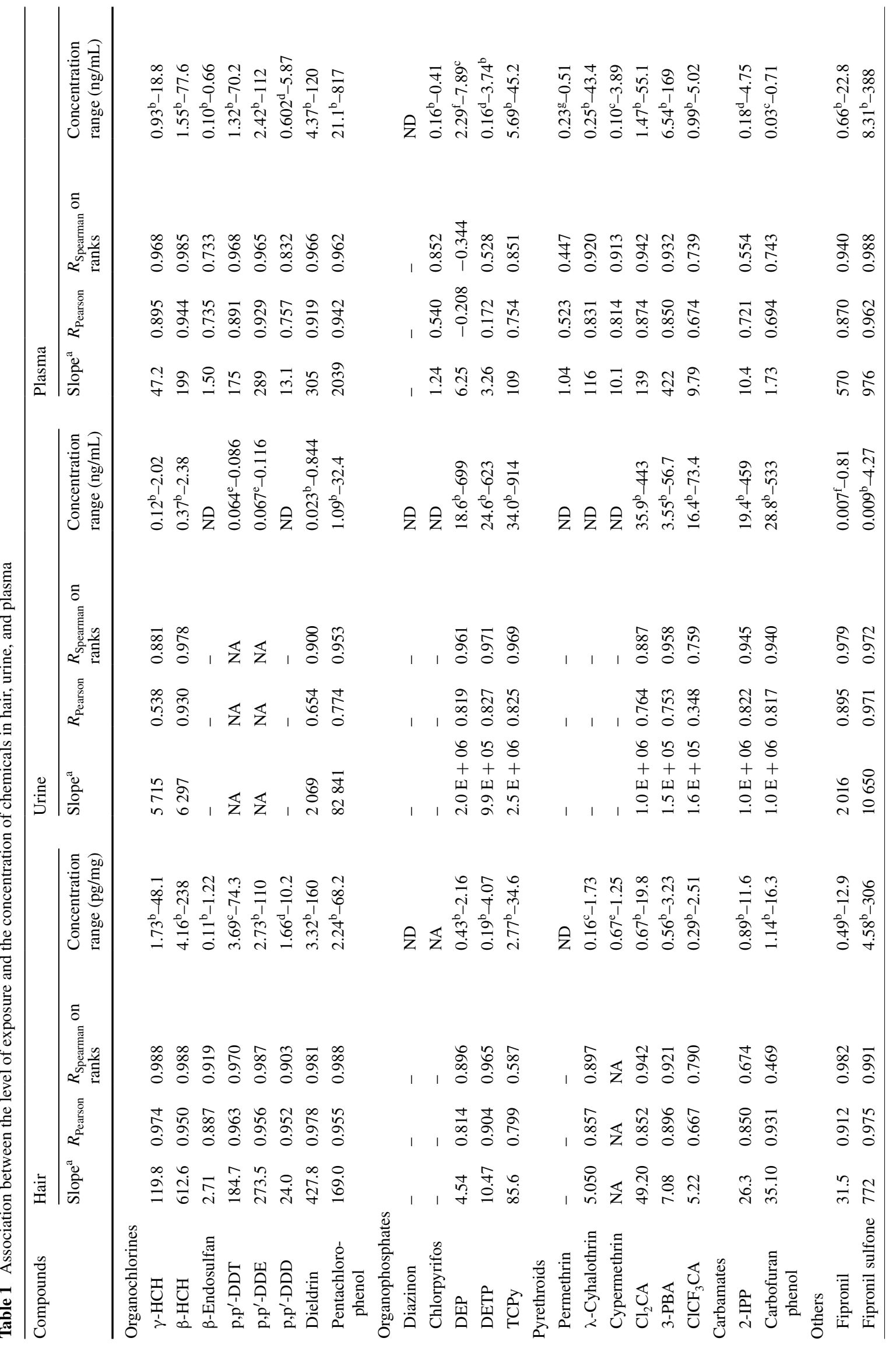




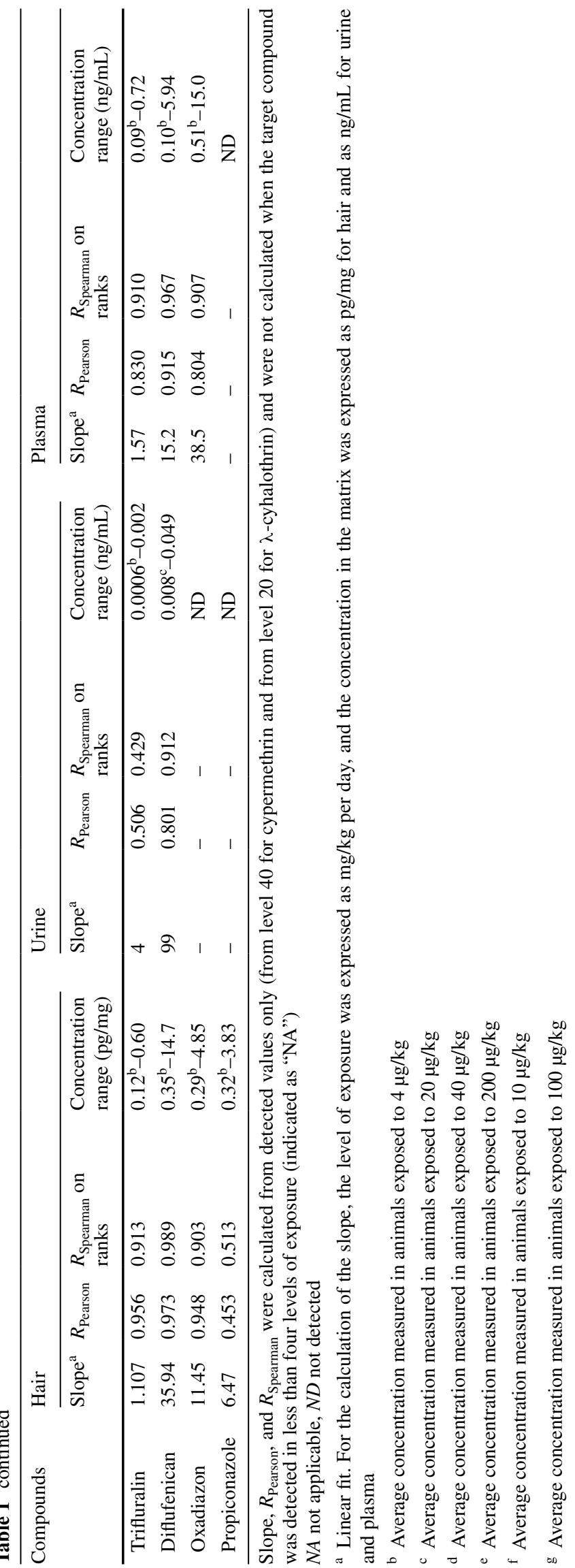



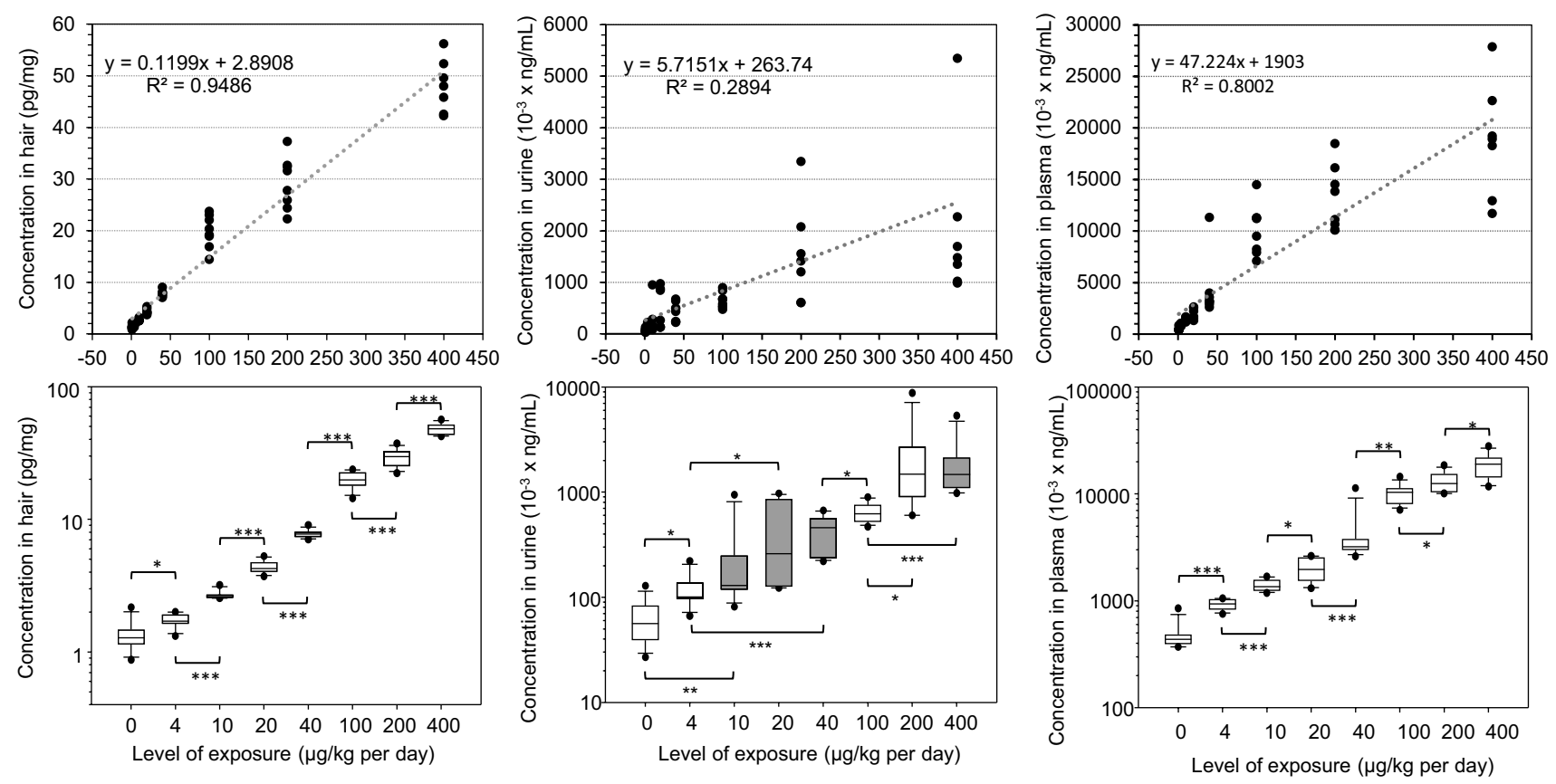

Fig. 1 Lindane ( $\gamma$-hexachlorohexane) concentrations in hair (left), urine (center), and plasma (right) of rats submitted to the different levels of exposure. The top panels present the proportional $x$-axes and provide details about each animal separately. In the urine chart, a point at $9000 \mathrm{pg} / \mathrm{mL}$ for the dosage of $200 \mu \mathrm{g} / \mathrm{kg}$ is not presented (out of scale) for better visibility. The bottom panels present box plots, with the bottom of the box representing the 25th quartile and the top of the box representing the 75th quartile. The line within the

between the different pesticides were also observed for urine and plasma.

\section{Organochlorines}

Organochlorines were detected in both hair and plasma at most of the levels of exposure, including the controls. In hair, exceptions were only observed for $\mathrm{p}, \mathrm{p}^{\prime}$ dichlorodiphenyldichloroethylene (, $\mathrm{p}^{\prime}$-DDE), which was not detected in controls, and $\mathrm{p}, \mathrm{p}^{\prime}$-DDT and $\mathrm{p}, \mathrm{p}^{\prime}$ dichlorodiphenyldichloroethane (, $\mathrm{p}^{\prime}$-DDD), which were only detected from levels of 20 and $40 \mu \mathrm{g} / \mathrm{kg}$ per day, respectively. In plasma, $\mathrm{p}, \mathrm{p}^{\prime}$-DDT was not detected in control animals, and its metabolite, p, $\mathrm{p}^{\prime}$-DDD, was detected from $40 \mu \mathrm{g} / \mathrm{kg}$. In contrast, urine presented the highest rate of "undetected" (Fig. 2), and only $\gamma-\mathrm{HCH}$ ( $\gamma$-hexachlorocyclohexane/lindane) and pentachlorophenol were detected in all the groups. In addition, $\mathrm{p}, \mathrm{p}^{\prime}$-DDT and $\mathrm{p}, \mathrm{p}^{\prime}$-DDE were only detected from the $200 \mu \mathrm{g} / \mathrm{kg}$ exposure level, and $\beta$-endosulfan and $\mathrm{p}, \mathrm{p}^{\prime}$-DDD were not detected at all in urine. As presented with the example of lindane ( $\gamma$-hexachlorohexane) (Fig. 1, box plot), significant inter-group differences in the concentration in animals' hair were observed for all of the organochlorines, with the box represents the median, and the whiskers reach at a maximum of 1.5 times the interquartile range. Circles represent outliers. White boxes mean that there is a significant difference $(p \leq 0.05)$ in chemical concentrations in hair with the preceding level of exposure. Light gray denotes that there is a marginally significant difference ( $p$ value slightly greater than 0.05 ), and dark gray means that there is no significant difference with the preceding level of exposure. $* p<0.05$, $* * p<0.01$, and $* * * p<0.001$

exception of $\beta$-endosulfan, in which a significant difference was observed between the high exposure groups only, and $\mathrm{p}, \mathrm{p}^{\prime}$-DDT and $\mathrm{p}, \mathrm{p}^{\prime}$-DDD, which were only detected from the exposure levels of 20 and $40 \mu \mathrm{g} / \mathrm{kg}$ per day, respectively (Fig. S2). In that regard, the behavior of organochlorines in plasma was very similar to what was observed in hair, although the inter-group difference was always slightly poorer in plasma. In urine, both the association between the level of exposure and the concentration in the matrix (assessed by $R_{\text {Pearson }}$ ) and the inter-group difference (assessed by $R_{\text {Spearman }}$ on ranks) were systematically lower than in hair and in plasma (Table 1). At equal levels of exposure, $\beta$-endosulfan presented the lowest concentration among all the organochlorines in both hair and plasma and was not detected in urine. The highest concentration was observed for $\beta-\mathrm{HCH}$ in hair and for pentachlorophenol in both urine and plasma.

\section{Organophosphates}

As previously mentioned in the "Materials and methods" section of this manuscript, the methodology allowed the quantification of parent organophosphates in urine and plasma, and only provided qualitative results in hair. 


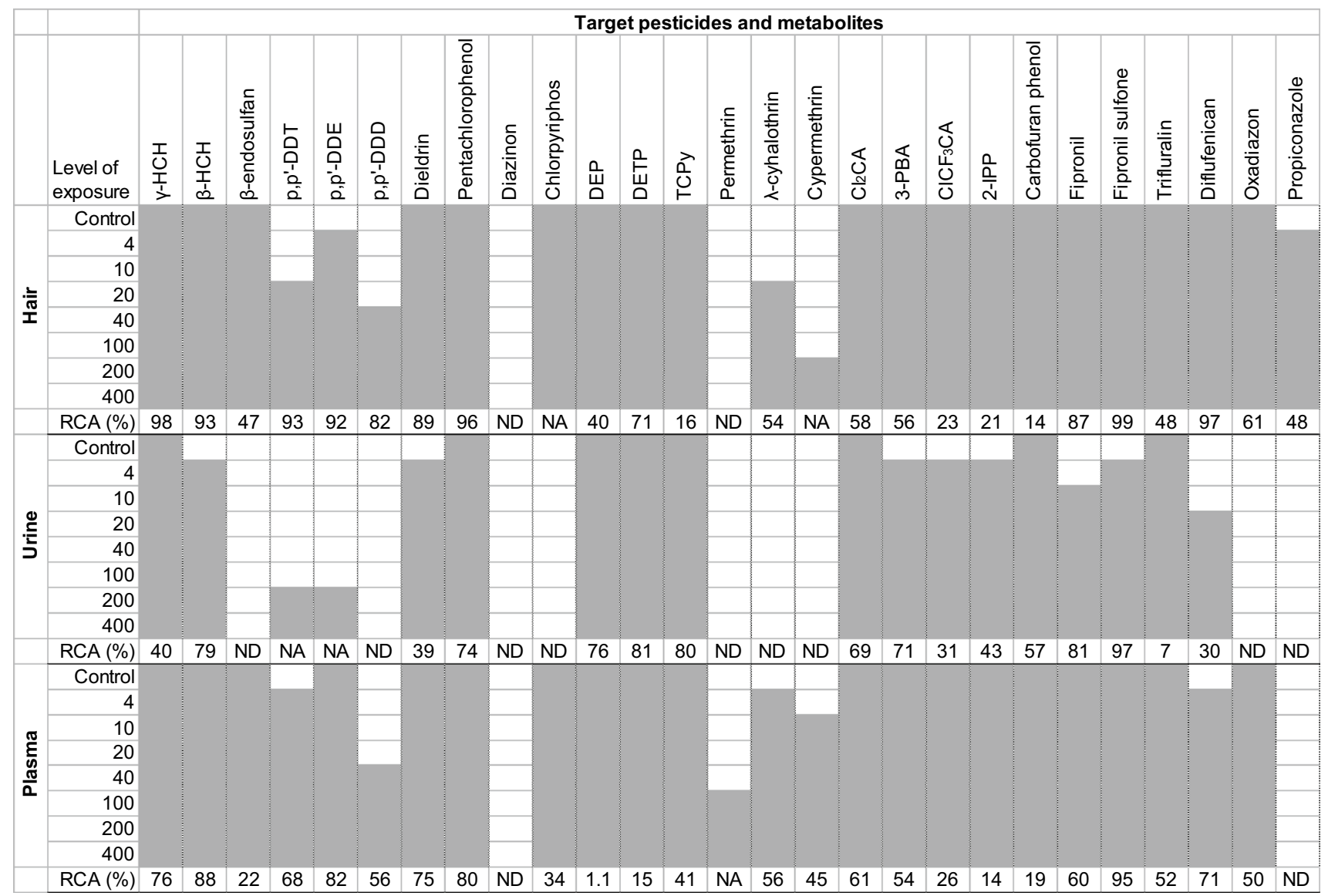

Fig. 2 Pesticides and metabolites detected in hair, urine, and plasma according to the animals' levels of exposure (gray cells denote positive detection) and reverse classification analysis (RCA) results,

No parent organophosphates were detected in urine. In plasma, among the two organophosphates administered to animals, only chlorpyrifos was detected, with a mean concentration ranging from $0.15 \pm 0.01 \mathrm{ng} / \mathrm{mL}$ in the control group to $0.41 \pm 0.09 \mathrm{ng} / \mathrm{mL}$ in the most exposed group. Metabolites of organophosphates (DEP, DETP, and TCPy: 3,5,6-trichloro-2-pyridinol) were detected in the three matrices, whatever the level of exposure, including the controls. A significant association was always observed between the metabolite concentration in the matrix and the level of exposure, with the exception of DEP and DETP in plasma (Table 1; Fig. S1). DETP, however, presented the best correlation between exposure and concentration in the matrix for both hair and urine (Table 1). As presented with the example of DEP (Fig. S1), inter-group differentiation based on metabolite concentration was best for urine, was acceptable for hair, and was quite poor for plasma, except for TCPy (Table 1; Figs. S2, S3, S4). Regarding the equivalent level of exposure, the highest concentration was observed for TCPy in the three matrices (Table 1). expressed as the percentage of correct classification based on the concentration in the matrix (Note: $N A$ not applicable, $N D$ not detected)

\section{Pyrethroids}

Although no parent pyrethroid was detected in urine, two out of the three pyrethroids to which animals were exposed were detected in hair and in plasma (Fig. 2). Cyhalothrin was detected from the $20 \mu \mathrm{g} / \mathrm{kg}$ exposure level in hair and from the $4 \mu \mathrm{g} / \mathrm{kg}$ exposure level in plasma. Cypermethrin was detected from the $200 \mu \mathrm{g} / \mathrm{kg}$ exposure level in hair and from the $10 \mu \mathrm{g} / \mathrm{kg}$ exposure level in plasma. Permethrin was only detected in plasma from $100 \mu \mathrm{g} / \mathrm{kg}$. At equal level of exposure, the $\lambda$-cyhalothrin concentration in plasma was approximately 10 times higher than cypermethrin and approximately 100 times higher than permethrin, which could explain why permethrin was not detected in hair. The three pyrethroid metabolites tested in the current study were detected in all the exposure groups in the three matrices, except for 3-phenoxybenzoic acid (3-PBA) and 3-(2-chloro-3,3,3-trifluoro-1-propenyl)-2,2-dimethylcyclopropane carboxylic acid $\left(\mathrm{ClCF}_{3} \mathrm{CA}\right)$ in the urine of the control animals. Urinary metabolites presented a weaker association with the level of exposure than the hair and plasma 


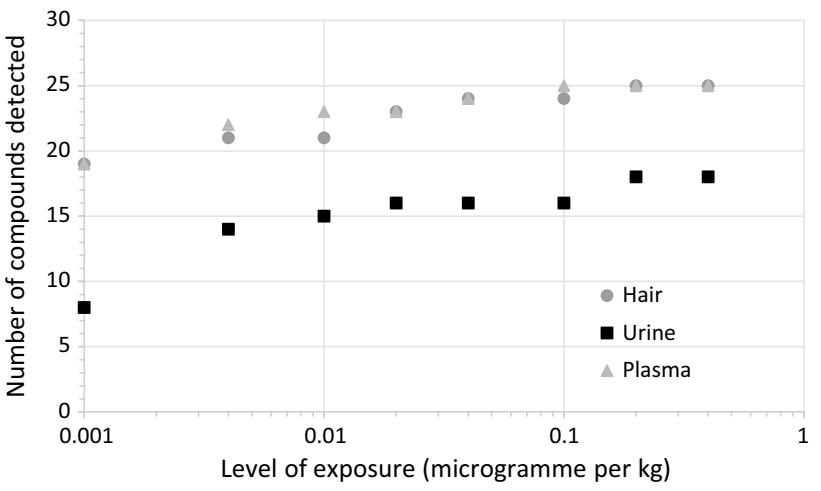

Fig. 3 Number of chemicals detected in hair, urine, and plasma for the different levels of exposure

concentrations $\left(R_{\text {Pearson }}\right)$ and comparable inter-groups differences. As for the metabolites of organophosphates, the urinary concentrations of 3-(2,2-dichlorovinyl)-2,2-dimethylcyclopropane-1-carboxylic acid $\left(\mathrm{Cl}_{2} \mathrm{CA}\right)$ and 3-PBA were not significantly different between the two highest levels of exposure. The highest concentration was observed for $\mathrm{Cl}_{2} \mathrm{CA}$ in hair and urine and for 3-PBA in plasma.

\section{Carbamates}

The two carbamate metabolites, 2-IPP and carbofuran phenol, were detected in all of the animal groups in the three matrices, except for 2-IPP in the urine of the controls animals. Inter-group differentiation was better in the highest exposure groups in hair, whereas it was better in the lowest concentration levels in urine, and it was quite poor in plasma, whatever the level of exposure (Table 1; Figs. S2, S3, S4).

\section{Other pesticides}

Fipronil and its metabolite fipronil sulfone were detected in all of the groups in hair and plasma, but the two compounds were not detected in the urine of the control animals, and fipronil was only detected from the $10 \mu \mathrm{g} / \mathrm{kg}$ exposure level. The two compounds presented good intergroup differentiation and a significant association between the concentration in the matrix and the level of exposure in the three matrices, although both $R_{\text {Pearson }}$ and $R_{\text {Spearman }}$ were higher for hair (Table 1). In the three matrices, the metabolite presented quite a higher concentration than the parent.

Trifluralin was detected in all of the groups, including controls, in hair, urine, and plasma, but the association between the concentration in the matrix and the level of exposure was quite better for hair and plasma than for urine. Similarly, the inter-group differentiation was quite easy when it was based on the hair and plasma concentrations, but was almost impossible with urine (Figs. S2, S3, S4). Diflufenican was detected in the hair of all of the animals, including the controls, from the $20 \mu \mathrm{g} / \mathrm{kg}$ exposure level in urine and from the first level of exposure in plasma. For diflufenican, the inter-group differentiation was best when it was based on the concentration in hair, followed by plasma, but it was rather poor when based on urine. Oxadiazon was detected in hair and plasma of all of the groups of exposure, with better inter-group differentiation in hair, but it was not detected in urine, whatever the level of exposure. Propiconazole was detected in the hair samples of all of the groups of exposure, but significant differences between adjacent groups were only observed between the three highest levels of exposure. Propiconazole was not detected in rat plasma and urine, whatever the level of exposure.

Adjusting the urinary concentration $(\mathrm{ng} / \mathrm{mL})$ with the volume of urine $(\mathrm{mL})$ to obtain the amount excreted over $24 \mathrm{~h}$ (ng) decreased the correlation with the level of exposure for all the chemicals except for TCPy $\left(R^{2}=0.7004\right.$ vs. 0.6802), DEP $\left(R^{2}=0.7069\right.$ vs. 0.6712), DETP $\left(R^{2}=0.7412\right.$ vs. 0.6845$), \mathrm{Cl}_{2} \mathrm{CA}\left(R^{2}=0.5956\right.$ vs. 0.5856$)$, 3-PBA $\left(R^{2}=0.6174\right.$ vs. 0.5676$)$, and 2-IPP $\left(R^{2}=0.6845\right.$ vs. 0.6750 ) for which the correlation was slightly increased. Similarly, adjusting the urinary concentration with the creatinine concentration had limited effects on both $R_{\text {Pearson }}$ and $R_{\text {Spearman }}$.

\section{Reverse classification analysis (RCA) and the number of detected pesticides}

The RCA scores based on pesticide concentration in the matrix are presented in Fig. 2. Hair provided the best RCA scores for 13 out of the 27 target compounds: all of the organochlorines, fipronil, fipronil sulfone, diflufenican, oxadiazon, and propiconazole. Hair also provided the lowest number of compounds that were never detected, whatever the level of exposure (two out of 27). Urine allowed reaching the highest RCA scores for eight compounds but provided a real advantage over hair and plasma only for four of them: DEP, TCPy, 2-IPP, and carbofuran phenol. Moreover, urine presented the highest number of undetected compounds (nine out of 27). Plasma provided the highest RCA scores for three compounds (i.e., chlorpyrifos, cyhalothrin, and trifluralin), although close RCA values were obtained with hair for cyhalothrin and trifluralin. In plasma, among the 27 target compounds, only propiconazole and diazinon were not detected. The number of target chemicals detected in the biological matrices increased with increasing exposure, but was always higher in hair and plasma than in urine (Fig. 3). In control animals, 19 compounds were detected in hair and in plasma, and eight were detected in urine. 


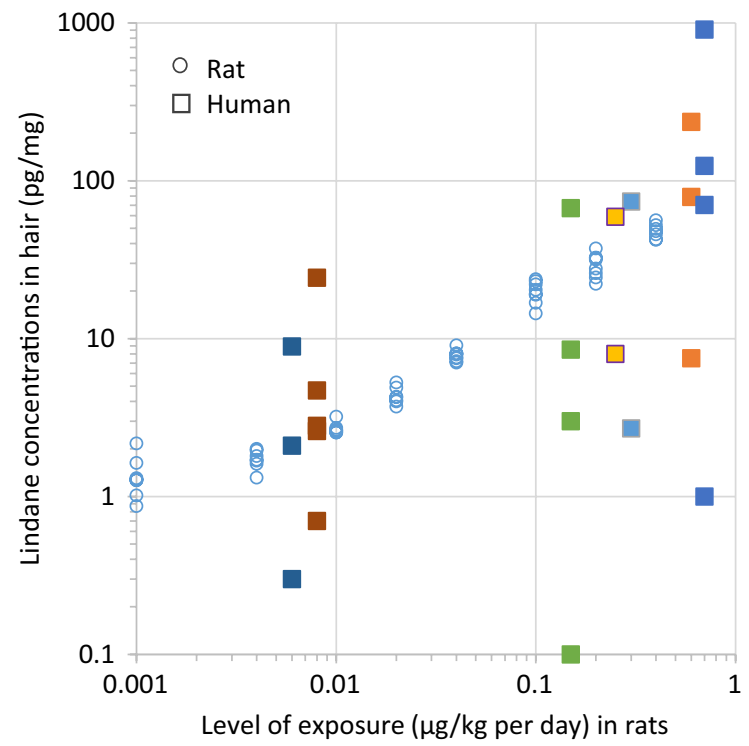

Fig. 4 (Left) lindane concentrations in rat hair (the current work) and in human hair (reported from the literature). (Right) 3-PBA concentration in rat urine (the current work) and in human urine (reported from the literature). The data that correspond to the rats (circles) are related to the levels of exposure presented on the $x$-axis. The data that correspond to humans (squares) are not related to the level of exposure and are situated close to similar range of concentration observed in rats for better visibility. For humans, the upper square represents the highest concentration detected, and the lower square represents the lowest concentration detected (Note: When the low-

\section{Pigmentation}

The influence of hair pigmentation on pesticide incorporation was assessed by comparing the concentration detected in white hair versus pigmented hair which were collected and analyzed separately from each animal. A slope close to the value of 1 indicated a poor influence of pigmentation on the compound concentration in hair (Table S2), as previously demonstrated in humans for other compounds (Appenzeller et al. 2007b). For the majority of the chemicals tested during the current study, pigmentation seemed to have a very limited effect on the concentration in hair, and only few compounds seemed to be pigmentation sensitive.

\section{Discussion}

The present results definitely demonstrate that the concentration of pesticides and their metabolites in hair is representative of the level of exposure. For all of the chemicals detected in hair, the association between the exposure and the concentration in hair was always significant, with the poorest $p$ value for $R_{\text {Pearson }}$ equal to 0.000274 (observed for propiconazole). For most compounds, the association

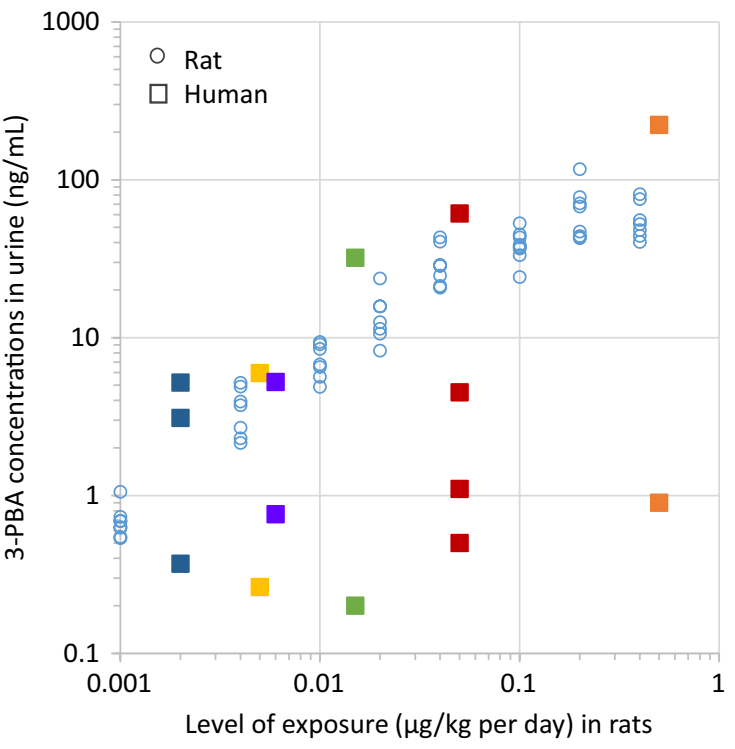

est value was not detected, it was replaced by a half limit of detection). An intermediate square represents the median or the mean value (when available); several intermediate squares correspond to several sub-populations. Data on humans were obtained from many publications (Attflied et al. 2014; Behrooz et al. 2012; Castorina et al. 2010; Covaci et al. 2002, 2008; McKelvey et al. 2013; Oulhote and Bouchard 2013; Salquebre et al. 2012; Tsatsakis et al. 2008a, b; Wielgomas 2013; Wielgomas et al. 2012; Zhang et al. 2007) and from personal data

between the exposure intensity and the concentration of chemicals in hair was stronger than or comparable to urine and plasma. Although the association was better for nonpolar compounds such as organochlorines, it also concerned polar compounds such as metabolites of organophosphate and pyrethroid pesticides.

The ability to differentiate animals from different groups of exposure or to reattribute individuals to their correct group of exposure based on pesticide concentration in a matrix depended on both the matrix and the chemical. Hair analysis proved to be a highly efficient method for organochlorines and other parent pesticides, and also provided relevant results for other compounds, including polar metabolites of organophosphate pesticides and pyrethroids. As expected, urine was best adapted for detecting polar chemicals such as organophosphates and pyrethroid metabolites, and it also allowed acceptable results to be reached for some parent compounds, including some organochlorine pesticides, although urine is generally not considered for their analysis (Centers for Diseases Control and Prevention 2009). Urine, however, presented the highest rate of non-detected compounds compared to the two other matrices. Plasma allowed relevant information to be obtained regarding exposure to most compounds, with the exception of dialkyl phosphates for which this matrix proved to be 
unsuitable. Although blood is considered to be the compartment of choice for organochlorine biomonitoring (Centers for Diseases Control and Prevention 2009), organochlorine concentration in hair presented a better association with exposure and allowed better $R_{\text {Pearson }}$ and $R_{\text {Spearman }}$ values and RCA scores to be reached than with plasma.

The differences in pesticide concentrations observed between the urine and the plasma compartments are likely attributable to pesticide metabolism and physicochemical properties. For instance, the low concentration of dialkyl phosphate in plasma, whatever the level of exposure of animals, could be explained by the fast metabolization of the parent compound ( $0.2 \mathrm{~h}$ for chlorpyrifos) (Timchalk et al. 2007) followed by rapid transfer of these highly hydrophilic metabolites to urine. In contrast, the hydrophobic character of some compounds such as pyrethroids and to a lesser extent organochlorines (Chata et al. 2016) may limit their transfer from plasma to urine. In that regard, hair appears to be less affected by this phenomenon and contains both hydrophilic and hydrophobic compounds.

It has to be noticed that the matrices investigated during the current study actually have different temporal windows of detection. An analysis of biological fluids (i.e., urine and blood) is rather representative of recent exposure, especially for chemicals with short life spans such as pyrethroid metabolites (3-PBA: half-life of $4-5 \mathrm{~h}$ in rat blood after oral dosing of pyrethroid) (Starr et al. 2012) and organophosphate metabolites (DEP: half-life of $0.2 \mathrm{~h}$ for $\alpha$-phase in rat blood after oral dosing of chlorpyrifos) (Timchalk et al. 2007). In contrast, hair is representative of periods covering weeks to months (Appenzeller and Tsatsakis 2012; Kintz et al. 2015). As a result, the protocol design of the current study, with an identical time lapse between gavage and fluid sampling for all of the animals, increased the apparent performances of urine and plasma analyses regarding representativeness of the exposure levels. In the case of random sampling time over the day, which is more representative of most epidemiological studies, the association between the pesticide concentration in the matrix and the level of exposure would have definitely been lower for plasma and urine because of intra-day variability, whereas it would not have affected results obtained from hair.

The presence of pesticides in controls was definitely not due to analytical contamination because the target compounds were always absent from the blank controls (solvent, extract without matrix) and was, therefore, really attributable to animal exposure (residual from the animal breeding center or the background level of exposure during experiments due to food, litter, or air), although these aspects were not further investigated during the current study. The presence of some chemicals in the control animals was not surprising because several compounds were reported to always be detected in humans (general population without specific exposure) (Salquebre et al. 2012) and also in control laboratory animals (Peiffer et al. 2013). The ability to document background exposure can be crucial, especially during experiments that are focused on low dose-related effects, because it may help to demonstrate (1) the exposure to the target compound(s) of controls that are supposedly not exposed and (2) the co-exposure to other chemicals that may induce combined effects. The two latter aspects may significantly bias interpretation of the results; therefore, these would need to be controlled. The current results of the study clearly highlight the relevance of hair, particularly over the choice of using urine for analysis for this purpose.

Although the influence of pigmentation on pesticide concentration in hair cannot be excluded, the current results demonstrated limited effects for most of the investigated pesticides. Because the range of concentrations of pollutants in hair within a population generally covers several orders of magnitudes (from the less exposed to the most exposed individuals), the differences observed during this study between samples of white and black hair are unlikely to induce significant misclassification of individuals according to their level of exposure. Moreover, the current situation (black hair with a high content of melanin vs. white hair, meaning absence of melanin) can be considered as an extreme scenario. The current study is therefore not representative of studies on humans, in which the percentage of individuals presenting totally non-pigmented hair is considered to be low, especially in children and pregnant women, which are favorite target populations for epidemiological studies focused on exposure and exposure-associated outcomes (Attflied et al. 2014; Burns et al. 2012; Castorina et al. 2010; Woodruff et al. 2011).

Although we conducted these experiments on an animal model, which is the only approach that allows for controlled exposure to pesticides, the purpose of the current study was definitely its transposition to human (with a view to classify individuals according to their level of exposure, or internal dose as final objective). We compared the pesticide concentration detected in biological matrices collected from the animals to data that were available in the literature for humans in the same matrices. Because information was not available for all of the pesticides tested in the current study, a comparison between humans and animals was focused on two chemicals for which large amounts of data are available in the literature. The two chemicals are lindane (organochlorine pesticide), which is to date among the most frequently analyzed chemicals in hair, and 3-PBA (pyrethroid metabolite), which is a frequently analyzed pesticide metabolites in urine. The range of concentrations of lindane in hair and 3-PBA in urine resulting from exposure of the animals was clearly comparable to concentration levels 
reported in the literature for humans (Fig. 4). We observed the lowest levels of lindane in human hair for inhabitants of Luxembourg $(n=14)$ (Salquebre et al. 2012) and Northern Poland $(n=40)$ (Wielgomas et al. 2012). The median (Luxembourg) and mean (Poland) concentration values were in the range of those detected in animals administered $4 \mu \mathrm{g} / \mathrm{kg}$ of lindane up to $20 \mu \mathrm{g} / \mathrm{kg}$, three times a week. The highest concentration of lindane in hair was observed for a Greek rural population, including possible occupational exposure (Tsatsakis et al. 2008a, b), with median concentration values above those detected in animals submitted to the highest level of exposure of our experiments $(400 \mu \mathrm{g} / \mathrm{kg})$. The lowest concentration of 3-PBA in human urine (Fig. 4) involved a French pregnant women (unpublished data), with a median value close to what was detected in animals exposed to $4 \mu \mathrm{g} / \mathrm{kg}$, three times a week. The highest concentration reported in the literature involved pregnant women living in the Salinas Valley in California (Castorina et al. 2010). The highest 3-PBA concentration in urine was above the concentration detected in the animals that were exposed to the highest level during our experiments.

In summary, the results of the current study demonstrate for the first time the significant association between the levels of exposure to pesticides and their resulting concentrations in hair. Even though we conducted this study on rats and used a limited number of pesticides, the hypothesis of similar behaviors for other chemicals and other species such as human seems to be reasonable. Regarding the transposition to humans, although pesticide concentration in hair does not yet allow for the determination of pesticide intake-at least because of interspecies differences-the current study still demonstrates that hair analysis allows reliable classification of individuals according to their respective level of exposure. This proof-of-concept, therefore, represents a step forward in considering hair analysis as a reliable tool to be used during epidemiological studies to investigate exposure-associated adverse health effects.

\footnotetext{
Acknowledgements The authors would like to thank Heather Farr for her support on editing the manuscript, and Jean-Charles Olry for providing technical support for animal maintenance. This study was carried out in the framework of the call for Research Project 2010 of the National Research Programme for Environmental and Occupational Health' (PNR-EST) of the French Agency for Food, Environmental and Occupational Health Safety (ANSES, Agence nationale de sécurité sanitaire, de l'alimentation, de l'environnement et du travail), with the financial support of the National Office for Water and Aquatic Environments (ONEMA, Office National de l'Eau et des Milieux Aquatiques) supporting the implementation of the Plan Ecophyto 2018, France. Radu-Corneliu Duca benefited from a postdoctoral grant from the Luxembourg National Research Fund (FNR, Fonds National de la Recherche) (AFR 1069412), Luxembourg. Caroline Chata benefited from a Ph.D. grant from the FNR (AFR 7009593), Luxembourg.
}

\section{Compliance with ethical standards}

Conflict of interest The authors confirm that they do not have any conflict of interest.

Open Access This article is distributed under the terms of the Creative Commons Attribution 4.0 International License (http://creativecommons.org/licenses/by/4.0/), which permits unrestricted use, distribution, and reproduction in any medium, provided you give appropriate credit to the original author(s) and the source, provide a link to the Creative Commons license, and indicate if changes were made.

\section{References}

Appenzeller BMR (2015) Hair analysis for the biomonitoring of human exposure to organic pollutants. In: Kintz P, Salomone A, Vincenti M (eds) Hair analysis in clinical and forensic toxicology. Elsevier, Academic Press, Amsterdam, London

Appenzeller BMR, Tsatsakis AM (2012) Hair analysis for biomonitoring of environmental and occupational exposure to organic pollutants: state of the art, critical review and future needs. Toxicol Lett 210(2):119-140

Appenzeller BMR, Agirman R, Neuberg P, Yegles M, Wennig R (2007a) Segmental determination of ethyl glucuronide in hair: a pilot study. Forensic Sci Int 173:87-92

Appenzeller BMR, Schuman M, Yegles M, Wennig R (2007b) Ethyl glucuronide concentration in hair is not influenced by pigmentation. Alcohol Alcohol 42(4):326-327

Attflied KR, Hughes MD, Spengler JD, Lu C (2014) Within- and between-child variation in repeated urinary pesticide metabolite measurements over a 1-year period. Environ Health Perspect 122(2):201-206

Aylward LL, Hays SM, Smolders R et al (2014) Sources of variability in biomarker concentrations. J Toxicol Environ Health B 17:45-61

Behrooz RD, Barghi M, Bahramifar N, Esmaili-Sari A (2012) Organochlorine contaminants in the hair of Iranian pregnant women. Chemosphere 86:235-241

Burns JS, Williams PL, Sergeyev O et al (2012) Serum concentrations of organochlorine pesticides and growth among Russian boys. Environ Health Perspect 120(2):303-308

Castorina R, Bradman A, Fenster L et al (2010) Comparison of current-use pesticide and other toxicant urinary metabolites levels among pregnant women in the CHAMACOS cohort and NHANES. Environ Health Perspect 118:856-863

Centers for Diseases Control and Prevention (2009) Fourth national report on human exposure to environmental chemicals. Department of Health and Human Services Centers for Disease Control and Prevention

Chata C, Hardy E, Grova N, Appenzeller BMR (2016) Influence of pesticide physicochemical properties on the association between plasma and hair concentration. Anal Bioanal Chem 408:3601-3612

Covaci A, de Boer J, Ryan JJ, Voorspoels S, Schepens P (2002) Distribution of organobrominated and organochlorinated contaminants in Belgian human adipose tissue. Environ Res Sect A $88: 210-218$

Covaci A, Hura C, Gheorghe A, Neels H, Dirtu AC (2008) Organochlorine contaminants in hair of adolescents from Iassy, Romania. Chemosphere 72(1):16-20. doi:10.1016/j. chemosphere.2008.02.058

Esteban M, Castaño A (2009) Non-invasive matrices in human biomonitoring: a review. Environ Int 35:438-449 
Grova N, Salquebre G, Appenzeller BMR (2013) Gas chromatography-tandem mass spectrometry analysis of 52 monohydroxylated metabolites of polycyclic aromatic hydrocarbons in hairs of rats after controlled exposure. Anal Bioanal Chem 405:8897-8911

Hardy EM, Duca RD, Salquebre G, Appenzeller BMR (2015) Multiresidue analysis of organic pollutants in hair and urine for matrices comparison. Forensic Sci Int 249C:6-19

Kavvalakis MP, Tzatzarakis MN, Alegakis AK, Vynias D, Tsakalof AK, Tsatsakis AM (2014) Development and application of GCMS method for monitoring of long-term exposure to the pesticide cypermethrin. Drug Test Anal 6:9-16. doi:10.1002/dta.1601

Kharbouche H, Steiner N, Morelato M et al (2010) Influence of ethanol dose and pigmentation on the incorporation of ethyl glucuronide into rat hair. Alcohol 44:507-514

Kintz P, Salomone A, Vincenti M (2015) Hair analysis in clinical and forensic toxicology. Academic Press, Elsevier, London, Amsterdam

Maravgakis G, Tzatzarakis MN, Alegakis AK, Stivaktakis PD, Tsatsakis AM (2012) Diethyl phosphates accumulation in rabbits' hair as an indicator of long term exposure to diazinon and chlorpyrifos. Forensic Sci Int 218(1-3):106-110. doi:10.1016/j. forsciint.2011.10.017

Margariti MG, Tsatsakis AM (2009) Assessment of long-term subacute exposure to dimethoate by hair analysis of dialkyl phosphates DMP and DMTP in exposed rabbits: the effects of dose, dose duration and hair colour. Environ Res 109(7):821-829. doi:10.1016/j.envres.2009.07.009

McKelvey W, Jacobson JB, Kass D et al (2013) Population-based biomonitoring of exposure to organophosphate and pyrethroid pesticides in New York City. Environ Health Perspect 121:1349-1356

Ntzani EE, Chondogiorgi M, Ntritsos G, Evangelou E, Tzoulaki I (2013) Literature review on epidemiological studies linking exposure to pesticides and health effects. EFSA Supporting Publications 2013:EN-497, p 159

Oulhote Y, Bouchard MF (2013) Urinary metabolites of organophosphate and pyrethroid pesticides and behavioral problems in Canadian children. Environ Health Perspect 121:1378-1384

Peiffer J, Cosnier F, Grova N et al (2013) Neurobehavioral toxicity of a repeated exposure (14 days) to the airborne polycyclic aromatic hydrocarbon fluorene in adult Wistar male rats. PLoS ONE 8(8):e71413
Salquebre G, Schummer C, Millet M, Briand O, Appenzeller BMR (2012) Multi-class pesticide analysis in human hair by gas chromatography tandem (triple quadrupole) mass spectrometry with solid phase microextraction and liquid injection. Anal Chim Acta 710:65-74

Starr MJ, Graham SE, Ross DG et al (2012) Environmentally relevant mixing ratios in cumulative assessments: a study of the kinetics of pyrethroids and their ester cleavage metabolites in blood and brain; and the effect of a pyrethroid mixture on the motor activity of rats. Toxicology 320:15-24

Timchalk C, Busby A, Campbell JA, Needham LL, Barr DB (2007) Comparative pharmacokinetics of the organophosphorus insecticide chlorpyrifos and its major metabolites diethylphosphate, diethylthiophosphate and 3,5,6-trichloro-2-pyridinol in the rat. Toxicology 237:145-157

Tsatsakis AM, Tzatzarakis MN, Tutudaki M (2008a) Pesticide levels in head hair samples of Cretan population as an indicator of present and past exposure. Forensic Sci Int 176(1):67-71. doi:10.1016/j.forsciint.2007.07.017

Tsatsakis AM, Tzatzarakis MN, Tutudaki M, Babatsikou F, Alegakis AK, Koutis C (2008b) Assessment of levels of organochlorine pesticides and their metabolites in the hair of a Greek rural human population. Hum Exp Toxicol 27(12):933-940. doi: $10.1177 / 0960327108102047$

Tutudaki M, Tsakalof AK, Tsatsakis AM (2003) Hair analysis used to assess chronic exposure to the organophosphate diazinon: a model study with rabbits. Hum Exp Toxicol 22:159-164

Wielgomas B (2013) Variability of urinary excretion of pyrethroid metabolites in seven persons over seven consecutive daysimplications for observational studies. Toxicol Lett 221:15-22

Wielgomas B, Czarnowski W, Jansen E (2012) Persistent organochlorine contaminants in hair samples of Northern Poland population, 1968-2009. Chemosphere 89:975-981

Woodruff TJ, Zota AR, Schwartz JM (2011) Environmental chemicals in pregnant women in the USA: NHANES 2003-2004. Environ Health Perspect 119(6):878-885

Zhang H, Chai Z, Sun H (2007) Human hair as a potential biomonitor for assessing persistent organic pollutants. Environ Int 33:685-693 